\title{
Kinematic Field Measurements During Orthogonal Cutting Tests via DIC with Double-frame Camera and Pulsed Laser Lighting
}

\author{
T. Baizeau ${ }^{1}$ (D ) S. Campocasso ${ }^{1,2}$ - G. Fromentin ${ }^{1} \cdot$ R. Besnard ${ }^{2}$
}

\begin{abstract}
The measurement of machined-part strain fields induced by the cutting process remains a challenge because of the presence of highly intensive and localised strains. In this study, a high-speed double-frame imaging device with pulsed laser lighting is used in order to obtain sharp and highly resolved images during orthogonal cutting tests performed in an aluminium alloy. The displacement fields are then measured using a global Q4-digital-image-correlation (DIC) method and several strategies, facilitating calculation of the total displacements due to the cut, along with the residual strains in the machined part. Numerical procedures are developed to manage the removed material that disturbs the DIC. An automatic primary shear angle detection procedure using DIC is also proposed. Five different markings, which are produced via chemical etching and micro blasting, are applied to the observed surfaces. Their effects on the kinematic fields and the uncertainties are then studied. Three surface parameters are proposed as indicators for determining the surface preparation suitability for the DIC. The repeatability of the kinematic fields induced during the cutting process is studied, because of the ease with which testing can be performed. Finally, the plastically deformed layer engendered by the cutting process is measured using the calculated residual strains.
\end{abstract}

T. Baizeau

thomas.baizeau@ensam.eu

1 Arts et Metiers ParisTech, LaBoMaP, Rue porte de Paris, 71250 Cluny, France

2 CEA, DAM, Valduc, 21120 Is-sur-Tille, France
Keywords Machining · Orthogonal cutting · Field measurement $\cdot$ Digital image correlation $\cdot$ High-speed imaging

\section{Introduction}

The lifetimes of machined parts subjected to fatigue or corrosive environments are heavily influenced by the surface integrity resulting from the employed cutting process $[1,2]$. Thus, prediction of the surface and sub-surface properties of mechanical components following cutting is one of the main issues affecting manufacturing research.

Therefore, in the past twenty years, a number of numerical simulations of cutting processes have been developed. With improved computer performance, inverse identification of material law parameters is now possible. However, validation of these simulations on a global scale remains excessively limited, and is achieved by comparing the cutting forces [3], the shear angle, or the chip morphology [4-6]. Post mortem data of the cut (shear angle, chip thickness) are primarily obtained by interrupting the process with a Quick Stop Device [7, 8]. This interruption is far from perfect; however, it is assumed that the tool removal has no influence on the obtained results. This time-consuming and low-repeatability method encourages exploration of process photography of the cut as an alternative technique, as proposed by Childs [9].

With the development of digital optical sensors, the number of reports on observations of in-situ cutting processes have increased. These observations were first conducted with low frame rates of 100 to $1000 \mathrm{fps}[5,10]$, and then with frame rates of up to $100 \mathrm{kHz}$ [11-15] using highspeed cameras. Classical high-speed cameras are primarily 
limited by exposure time, which is typically higher than 1 $\mu$ s. Low sensor sensitivity can also be detrimental to the image quality, because of the high lighting power density required for a small-scene observation [15]. Pulsed laser lighting combined with double-frame imaging is a common means of performing very-high-speed imaging, as it can be used for fluid mechanics, ballistics, or pyrotechnics experiments. Research interest regarding these devices is focused on the effective exposure determined by the laser pulse width, which has a value of a few nanoseconds, and the high lighting power density. Thus, sharp images of very short-term phenomena can be recorded. In 2008, this technique was successfully applied to cutting experiments by Hijazi and Madhavan [12] who synchronized four of these devices (laser + charge-coupled-device (CCD) camera), to obtain eight pictures with an inter-framing time of $10 \mu \mathrm{s}$ (100 kfps).

In parallel, the development of computerised image processing for studies of fluids (known as particle image velocimetry (PIV)) or solid mechanics (referred to as digital image correlation (DIC)) has facilitated measurement of displacement and strain fields. These processing techniques can be employed in one or two ways: local (PIV and DIC) or global (DIC only). For local processing, independent interrogation windows that can independently deform [16] and even overlap are followed. The displacement is applied at the centre of the interrogation window [17]. In the second approach, the calculation is conducted over the entire region of interest (ROI) [18] using a continuous mesh. Therefore, this method can be directly linked to finite element simulations.

For both DIC approaches, the estimated displacement field $\underline{U}$ must minimise the residual grey-level function $\tau$ presented in Eq (1) between two images $I$ and $J$ of the same scene taken at different times. The main specificity of global DIC is that the minimisation of the $\tau$ function is processed over the entire ROI such that

$\tau=\int_{R O I}[I(\underline{x})-J(\underline{x}+\underline{U}(\underline{x}))]^{2} d \underline{x}$.

In the so called Q4-DIC method, $\underline{U}$ is expressed as a function of the node displacements $\underline{U}_{n}$ using Q4P1 shape functions $\psi_{n}^{Q 4 P 1}$ on each finite element (called zones of interest (ZOIs)) as proposed by Besnard [19], where

$\underline{U}=\sum_{n} \underline{U}_{n} \cdot \psi_{n}^{Q 4 P 1}(\underline{x})$.

The use of a finite element (FE) mesh ensures continuity of the displacements over the entire ROI, precluding any overlapping or discontinuity, which could appear with local DIC or PIV approaches. For complex fields in solid mechanics, the global approach appears to have higher accuracy [20] because of the continuity of the mesh imposed by this method.
In order to determine the strains inside the work material, different markings have been applied to the surfaces prepared for observation: paint [20], grids [11, 21, 22], lines [14], or directly via microstructural observation after etching [12, 13, 15]. The laser-induced fluorescence (LIF) technique is currently being employed for PIV in the field of fluid mechanics [23]. However, LIF is not widely used in solid mechanics research as the observed surfaces generally exhibit homogeneous roughness. Nevertheless, an application of this technique to a study on the punching process should be mentioned here [24].

As regards machining studies, both PIV [12, 13, 15] and DIC $[11,25]$ methods have been employed. However, those studies primarily focused on primary shear plane analysis and used local approaches to achieve image correlation.

The present article presents an intensive study of the possibilities and accuracy of DIC during orthogonal cutting tests conducted on EN AW-7020-T6 aluminium alloy. The experimental set-up shown in Fig. 1 is first described in detail. Then, the a priori performance is investigated. To achieve this, five surface preparations are compared in terms of the corresponding DIC uncertainty. The repeatability and homogeneity for each surface preparation over the specimen surface are determined; hence, the most appropriate surface texture for DIC is selected. Numerical procedures for analysing the primary shear zone and for post-cutting residual strain field measurements are shown. The expression "residual strain" refers to the combination of the irreversible plastic strain and the residual elastic strain engendered by the residual stress state of the material. The kinematic fields engendered by the cutting process are determined. Because of the ease with which testing is performed, the repeatability of the cutting process is studied from the DIC perspective.

\section{Experimental Setup}

\section{Imaging Device, Settings, and Calibration}

The imaging device included a scalable complementary metal-oxide-semiconductor (sCMOS) PCO edge 5.5 camera

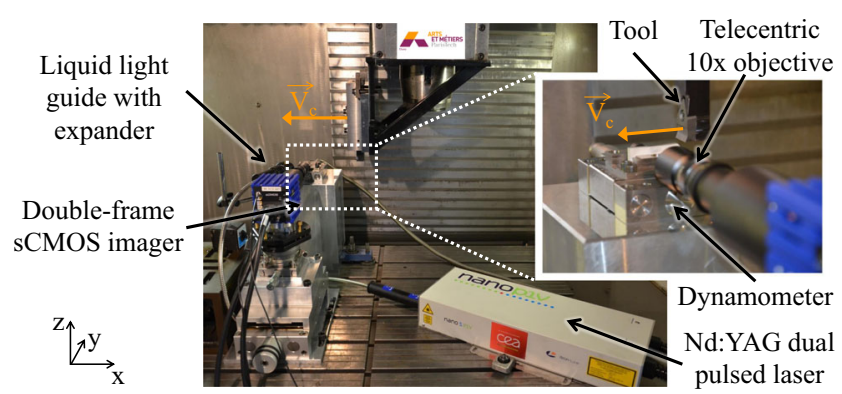

Fig. 1 Experimental setup for orthogonal cutting testing 
and a Litron Nano-S Nd:YAG laser (30-mJ specified energy), which was integrated by LaVision. The camera was used in double-frame mode, allowing very low interframing times (down to $120 \mathrm{~ns}$ ). The resolution of the sCMOS sensor was $2560 \times 2160$ pixels with a size of approximately $16.6 \times 14 \mathrm{~mm}$, and the dynamic range was 16 bits (65536 grey levels).

The laser head generated two pulses with a 532-nm wavelength, corresponding to the second harmonic of the $\mathrm{Nd}$ :YAG laser. The pulse duration was 5-8 ns, which corresponds to the effective exposure time as the testing machine was fully sealed. After passing through an optical collimator and a liquid light guide, the laser beam was split by an expander yielding a light spot of approximately 30 $\mathrm{mm}$ in diameter that allowed even illumination of the entire scene.

Magnification was achieved using a Mitutoyo ML 10× telecentric microscope objective (Ref. 375.039, numerical aperture (NA): 0.21 ) with a 51-mm nominal working distance. The telecentric objective was chosen so as to limit the image distortion. As the objective was used alone in order to maximise the NA, the magnification was determined by the distance to the sensor and calibration was required.

Image scaling was achieved using a Brinell indentation on a polished steel specimen the diameter of which (0.832 mm) was previously measured using an Olympus BX51M optical microscope. A $0.66-\mu \mathrm{m} / \mathrm{px}$ scale was obtained because of the set distance between the objective and the camera sensor, which corresponded to a field size of $1.7 \times 1.4 \mathrm{~mm}$. This distance was fixed during all experiments in order to maintain constant magnification.

The polished indented specimen was also used to calibrate the laser intensity. Indeed, the camera sensor could be damaged by the direct reflection of the laser beam by the bright, freshly generated surfaces (chips and burrs). During illumination of the spherical Brinell indent, an angular portion of the sphere directly reflected light onto the optical sensor. Thus, the laser power was adjusted at the opticalsensor saturation limit (shown in red in Fig. 2) finally corresponding to approximately $13 \%$ of the maximum power.

As the lighting was calibrated using the polished indented specimen shown in Fig. 2, only a few thousand of the 65536 grey levels were exploited using the machined specimens. Therefore, all the exposed pictures in this article are 8-bit-lightened and compressed versions of the 16-bit full-definition pictures used for the DIC.

\section{Cutting Test Configuration}

As shown in Fig. 1, a special experimental set-up for orthogonal cutting was integrated into a DMG DMC85V computer-numerical-control (CNC) milling machine. The

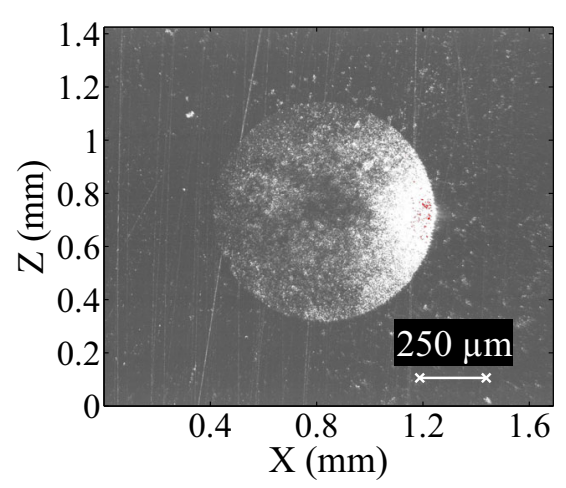

Fig. 2 Polished and indented specimen used for scaling and laser intensity tuning

tool was clamped in a square fixed on the spindle nose. A given cutting speed was achieved by translating the slide along the $\mathrm{x}$-axis in the negative direction (from right-toleft). The uncut chip thickness was adjusted by tuning the $\mathrm{z}$-axis of the milling machine. A riser block clamped on the machine table was used to level the sample with the imaging device optical axis. This planing configuration offers the advantage of zero motion between the sample and optical device assuring a low level of blurring.

The machined specimens used in this study were $40 \times$ $15 \times 4 \mathrm{~mm}^{3}$ cuboid specimens that were roughly produced via milling from wrought aluminium alloy blanks (EN AW7020-T6). Then, the observation surface on the specimen side was treated in order to obtain an appropriate roughness for the DIC, as detailed below ("Surface Preparation of Machined Specimens").

The orthogonal cutting configuration was obtained by planing the top of the specimen with a tungsten carbide insert (rake angle $\gamma=20^{\circ}$; clearance angle $\alpha=5^{\circ}$; ISO $\mathrm{K} 20$ grade), which was specially ground from ARNO ProfilCut inserts. The tool edge radius was measured to be $r_{n}$ $=3.9 \pm 1.5 \mu \mathrm{m}$ using a Somicronic Surfascan mechanical profilometer. The observed side of the insert that was first plane ground parallel to the working plane $P_{f}$, was positioned $0.02 \mathrm{~mm}$ in front of the specimen (y-direction) and remained in the depth of field.

The maximum achievable cutting speed was $120 \mathrm{~m} / \mathrm{min}$, which was the highest feasible speed for the linear motor drive of the machine employed in this study. In order to reduce the dynamic loads on the imaging device before the image acquisition, the machine acceleration was limited to 8 $\mathrm{m} / \mathrm{s}^{2}$ (instead of $14 \mathrm{~m} / \mathrm{s}^{2}$ ) using motion-synchronous actions available for the Siemens 840D numerical control (NC) used in this study.

A Kistler 9119AA2 piezoelectric dynamometer with a 5019A charge amplifier was used to measure the forces applied on the workpiece during the cutting test. 
Fig. 3 Schematic of acquisition and synchronisation device

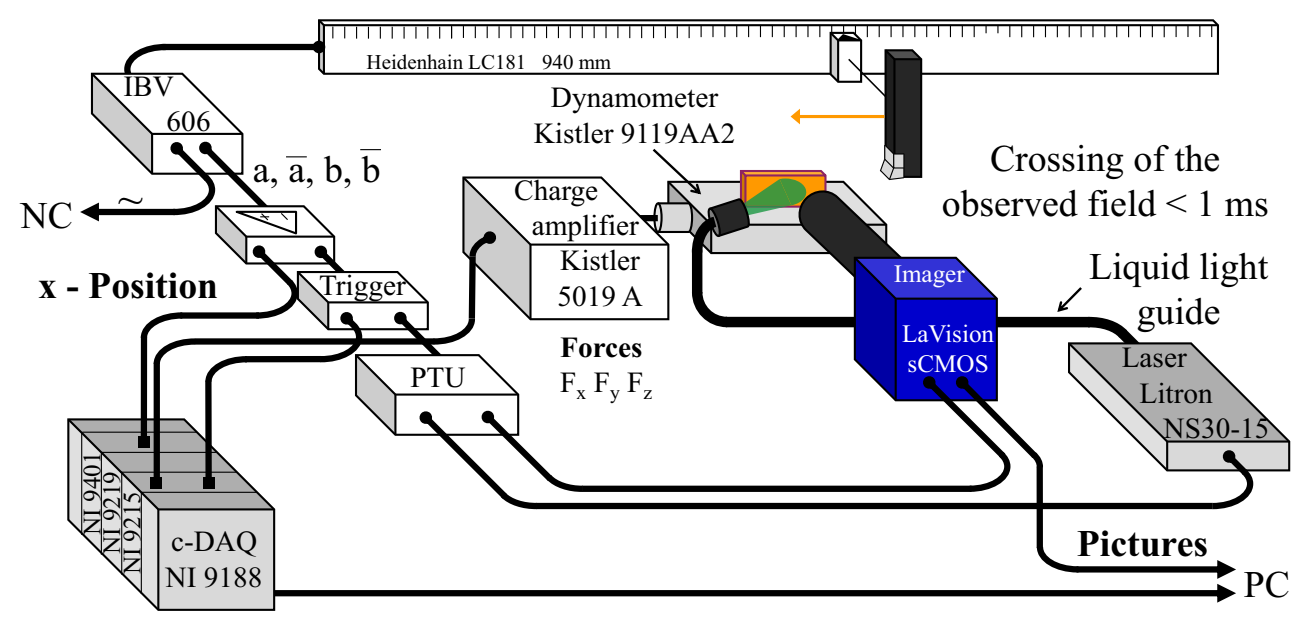

\section{Acquisition Device Synchronisation}

As a result of the small observation field and the high cutting speed, the tool passed through the field of view in less than $1 \mathrm{~ms}$ at $120 \mathrm{~m} / \mathrm{min}$; thus, reliable synchronisation between the tool position and the imaging system was required. The imaging device was triggered by a dedicated system, which was based on signal counting of the linear encoder of the machine-tool x-axis. A Heidenhain IBV606 splitter box was positioned between the Heidenhain LC181 encoder and the NC. This splitter box duplicated the position sinusoidal signals $a$ and $b$ and their conjugates (16- $\mu \mathrm{m}$ step), converting them into transistor-transistor logic (TTL) signals and performing an $\times 2$ interpolation as shown in Fig. 3. The interpolated signals were sent to a high-speed 32-bit quadrature counter chip used in the $\times 4$ mode. Thus, a 2- $\mu \mathrm{m} x$-position resolution was achieved. It should be noted that the triggering-device counting rate was $16 \mathrm{GHz}$, which allowed theoretical cutting speeds of more than 7000 $\mathrm{m} / \mathrm{min}$. In contrast, this speed was limited to $90 \mathrm{~m} / \mathrm{min}$ when these tests were first introduced, and therefore previous experiments were conducted under that limit [26, 27].

Synchronisation was achieved between the camera and the laser using a programmable timing unit (PTU) and LaVision DaVis software, ensuring that each frame of the pair was illuminated by one laser flash only.

A National Instrument cDAQ-9188 chassis, with a NI 9215 analogue input module and a NI 9401 digital counting data acquisition module, were used for synchronous recording of the following signals with the same clock time: the three components of the cutting force, the x-position, and the PTU triggering signal.

For each cutting test, double frames were acquired before, during, and after the cut as shown schematically in Fig. 4. The frame pairs were denoted Pi with $i=\{0, \ldots$, 4\}. The first frame for each pair was denoted f0, while the second frame was f1. By applying the DIC to the frame combinations, the chip formation, the total or residual strains, and the DIC performances could be analysed as follows:

- "During cutting" frame f1 was compared to "during cutting" frame f0 (P2f1/P2f0), allowing calculation of the kinematic field during the chip formation (primary shearing);

- The "during cutting" frame pair was compared to the "pre-cutting" frame pair (P2\{f0, f1\}/P1\{f0, f1\}), allowing calculation of the total strain field in the subsurface due to the cutting process;

- The "post-cutting" frame pair was compared to the "pre-cutting" frame pair (P3\{f0, f1\}/P1\{f0, f1\}), in order to evaluate the residual strains only;

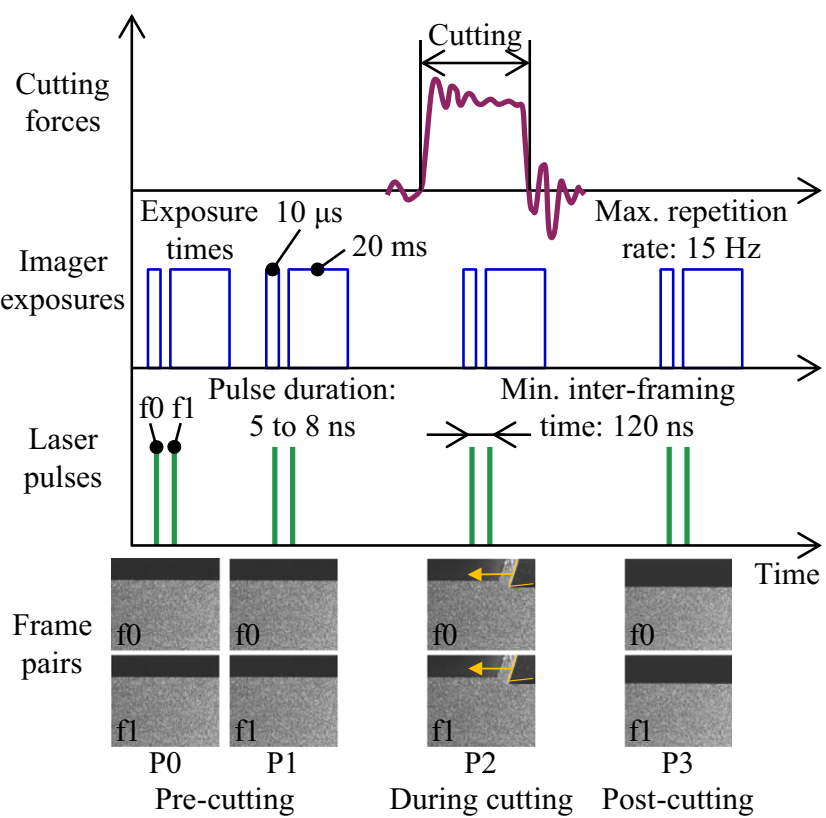

Fig. 4 Schematic of experimental time chart 
- The "pre-cutting" frame pair was compared to another "pre-cutting" frame pair (P1\{f0, f1 $\} / \mathrm{P} 0\{\mathrm{f} 0, \mathrm{f} 1\})$, in order to evaluate the entire experimental uncertainty of the displacement measurement.

As the exposure times of the two frames were inequivalent, f0 was always used for analysis of the "during/precutting" pairs (P2f0/P1f0) and the "post-/pre-cutting" pairs (P3f0/P1f0). This approach was employed in order to reduce the noise induced by the ambient light, which was non-zero, despite the careful caulking of the milling machine.

\section{A Priori DIC Performance}

The aim of this section is to evaluate, a priori, the performance of five surface preparation treatments in terms of DIC. The surface parameters are analysed with respect to the quality and uncertainty, which can be determined for each marking by analysing reference images (or "precutting" images). First, criteria from the software are employed; then, a metrological investigation of the DIC uncertainty is conducted.

\section{Surface Preparation of Machined Specimens}

In this study, the observed sides of the specimens were first mirror polished. Then, one specimen was plunged into a Keller etchant bath for $30 \mathrm{~s}$. The other specimens were blasted with glass micro-beads having diameters of 50 to $100 \mu \mathrm{m}$, under several blasting pressures: 1, 2, 3, or 5 bar. The resultant surface textures are shown in Fig. 5. It should be noted that a similar roughness to micro-bead blasting has been obtained via electro-discharge machining (EDM) of a hardened steel [26].

As the grey-level textures of the images are given by the diffuse reflection of the laser on the rough specimen surfaces, the observed surface roughness was measured using a Veeco Wyko NT1100 interferometer. For each marking type, five different $1.2 \times 1.2 \mathrm{~mm}^{2}$ areas were measured and the following roughness parameters were chosen as being representative of the DIC performance:

- The peak density $S_{p d}$, which is linked to the marking fineness;

- The mean peak curvature $S_{p c}$, which is linked to the grey level fluctuation.

The average values of these two parameters, together with the arithmetical mean height $S_{a}$ for the five measurements of each marking are given in the Table 1 .

In order to evaluate the impact of the roughness on the DIC, the correlation radius criterion defined in [19] was applied.

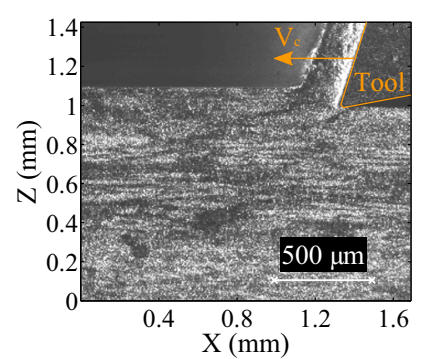

(a)

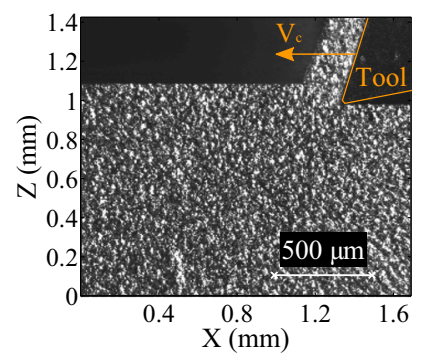

(c)

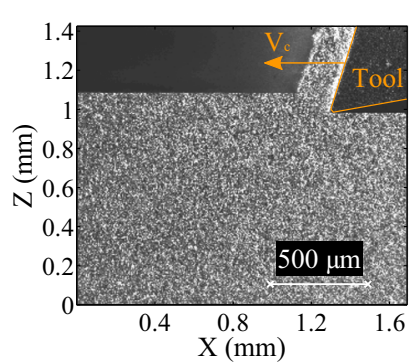

(b)

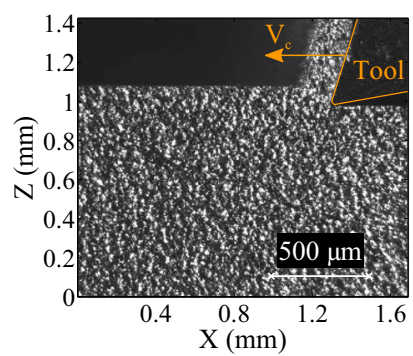

(d)
Fig. 5 Examples of surface preparations: a Etched or blasted at b 1, c 2 , or d 5 bar

This criterion represents the percentage of mesh elements, called ZOIs, that contain sufficient grey-level fluctuations to perform the correlation. The minimal element size $l$ of the employable ZOIs was determined using this criterion. Table 2 clearly shows that for all surface textures, a minimal ZOI size of ten pixels was required.

In order to conduct an identical analysis for every preparation type, an $l=12 \mathrm{px}$ element size $(7.9 \mu \mathrm{m})$ was employed for all the subsequent investigations.

\section{Measurement Uncertainties}

From 30 reference frame pairs $(\operatorname{RP} p, p=\{1, \ldots, 30\})$ of each marking, the displacement field between the frames $\mathrm{f} 0$ of the pair RP1 (RP1f0) and the $p$-th (RPpf0) of the 29 other pairs $\underline{U}_{p / 1}$ was calculated for $l=12 \mathrm{px}(196 \times 232$ elements $)$. Then, the uncertainties were estimated by calculating the standard deviation of each node displacement $U_{n}$ for either the $\mathrm{X}$ - or $\mathrm{z}$-direction as shown in Eq (3). Therefore, each

Table 1 Influence of micro-blasting pressure or etching on surface texture

\begin{tabular}{llll}
\hline $\begin{array}{l}\text { Surface preparation } \\
\text { (bar) }\end{array}$ & $\begin{array}{l}S_{p d} \\
\left(\text { peaks } / \mathrm{mm}^{2}\right)\end{array}$ & $\begin{array}{l}S_{p c} \\
(1 / \mathrm{mm})\end{array}$ & $\begin{array}{l}S_{a} \\
(\mu \mathrm{m})\end{array}$ \\
\hline 1 & 1230 & 107 & 0.31 \\
2 & 440 & 181 & 0.67 \\
3 & 476 & 343 & 1.06 \\
5 & 430 & 447 & 1.46 \\
etched & 94.3 & 34.2 & 0.03 \\
\hline
\end{tabular}


Table 2 Number of ZOI satisfying correlation radius criterion for three element sizes $l: 6,8$, and 10 pixels

\begin{tabular}{llcl}
\hline Surface preparation & \multicolumn{2}{l}{$\%$ of validated ZOI vs $l$ : } \\
\cline { 2 - 4 } (bar) & $6 \mathrm{px}$ & $8 \mathrm{px}$ & $10 \mathrm{px}$ \\
\hline 1 & 20.5 & 87.0 & 99.4 \\
2 & 20.6 & 85.9 & 99.0 \\
3 & 22.3 & 84.0 & 98.0 \\
5 & 22.9 & 83.4 & 97.6 \\
etched & 22.7 & 88.3 & 99.5 \\
\hline
\end{tabular}

node (acting as a displacement probe) was qualified in terms of accuracy. Note that the standard deviations were considered because the mean values were equal and represented rigid body motion of the entire observed area.

$\sigma_{U_{x, z} n}=\sqrt{\frac{1}{P-1} \sum_{p=2}^{P=30}\left(U_{x, z p / 1 n}-\bar{U}_{x, z n}\right) .}$

Hence, uncertainty maps were obtained for each marking as shown in Fig. 6(a) and (b) for the polished-etched sample and the most finely micro-blasted specimen (1 bar), respectively. The sample subjected to the micro-blasted preparation treatment yielded a homogeneous error distribution on the sensor; whereas, the polished-etched specimen exhibited strips of uncertainty levels. Comparison of those strips with the reference image indicates a match with the exposed grains that were deformed during the currying process of the blank. Based on these observations, the etched samples are not preferable for the DIC measurements because of the inhomogeneity of the uncertainty induced by the surface preparation.

Next, the frequency distributions of the uncertainties over the mesh $(196 \times 232)$ were compared and fit to a theoretical standard distribution according to a $95 \%$ confidence Pearson's $\chi^{2}$ test. Hence, the markings could be compared

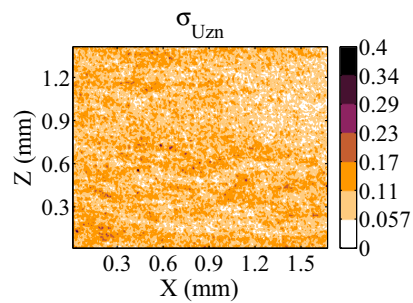

(a)

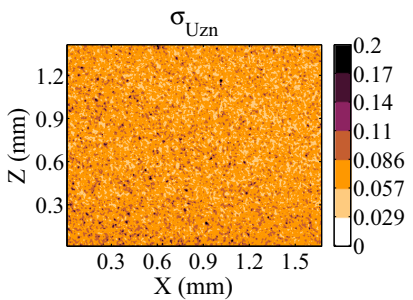

(b)
Fig. 6 Uncertainty maps in z-direction for two specimens subjected to different surface preparations: a Etched; b Blasted under 1-bar pressure against the average uncertainties, $\bar{\sigma}_{U_{x, z}}$, and the standard deviations of the uncertainties on the sensor, $\sigma_{\sigma_{U_{x, z}}}$, where

$\bar{\sigma}_{U_{x, z}}=\frac{1}{N} \sum_{n=1}^{N} \sigma_{U_{x, z} n}$,

$\sigma_{\sigma_{U_{x, z}}}=\sqrt{\frac{1}{N-1} \sum_{n=1}^{N}\left(\sigma_{U_{x, z} n}-\bar{\sigma}_{U_{x, z}}\right)}$.

The average uncertainty represents the ability of the complete measurement device to record the kinematic fields precisely. All sources of noise were taken into consideration: the milling-machine vibrations, the surface preparation techniques, the optics, the image sensor, and the digital image correlation. Note that the standard deviation of the uncertainty represents the homogeneity of the accuracy over the image sensor. Ultimately, the surface preparation yielding the highest homogeneity over the sensor and the lowest average uncertainty is preferred. That measurement corresponds to the highest possible precision over the entire sensor. As summarised in Table 3, the 1-bar blasted surface appears to correspond to the optimal preparation in terms of DIC.

The uncertainty analysis was also performed by considering the DIC between f0 and $\mathrm{f} 1$ for each of the 30 picture pairs. The obtained uncertainty levels are in the same order as those previously obtained between the RP $p \mathrm{f} 0, \mathrm{p}$ $=\{2, \ldots, 30\}$, and the RP1f0 frames. Therefore, the two laser heads, which ensured the lighting, were appropriately calibrated, because the difference between the Ppf0/P1f0 frames (same laser head) was not greater than that between the RP $p f 1 / \mathrm{RP} p \mathrm{f} 0$ frames (different laser heads).

Through this preliminary analysis of the DIC performance and also as a result of the complete experimental set-up, it has been shown that the etched preparation induces heterogeneity of the uncertainties, unlike the micro-blasted surfaces. The minimal element size (12 px) and the uncertainties were computed indicating that the lower microblasting pressure (1 bar) seems to be more appropriate

Table 3 Uncertainty analysis of specimen preparations based on 30 images of the same area, analysed using DIC

\begin{tabular}{lll}
\hline $\begin{array}{l}\text { Surface } \\
\text { preparation } \\
(\text { bar })\end{array}$ & $\begin{array}{l}\text { DIC } \\
\text { mean uncertainty } \\
(\mu \mathrm{m})\end{array}$ & $\begin{array}{l}\text { Standard } \\
\text { deviation } \\
(\mu \mathrm{m})\end{array}$ \\
\hline 1 & 0.088 & 0.025 \\
2 & 0.101 & 0.035 \\
3 & 0.120 & 0.041 \\
5 & 0.111 & 0.037 \\
etched & 0.119 & 0.039 \\
\hline
\end{tabular}


for the DIC calculation. Furthermore, because of the time required to compute the DIC on the 30 heavy pictures $(2560$ $\times 2160 \mathrm{px}$, 16-bit encoded, approximately $30 \mathrm{~min}$ ), this prior analysis was performed once for the entire image sensor. Finally, using the "pre-cutting" picture pairs (P0 and P1), which were obtained before the cut, it was possible to determine the order of the DIC accuracy. Therefore, the procedure could be shortened to $1 \mathrm{~min}$. This shorter procedure could also be applied to determine the optimal lighting configuration because of the high sensitivity of the DIC uncertainty to the grey levels.

\section{Application of DIC Strategies and Numerical Methods for Double-frame Imaging to Orthogonal Cutting}

The aim of this section is to present two numerical methods developed for primary shear angle detection and residual strain field calculation.

\section{Automatic Primary Shear Angle Measurement}

The picture pair taken during the cut (P2f0/P2f1) allowed analysis of the chip formation. The inter-framing time was set to $15 \mu \mathrm{s}$, corresponding to $23-\mu \mathrm{m}$ displacement of the tool in the cutting direction. The tool motion constrained the movement of the primary shear plane (PSP). By applying DIC near the cutting tool tip, the kinematic fields in the primary shear zone were measured as shown in Fig. 7 through a magnitude representation of the field.

A 2- $\mu \mathrm{m}$ threshold was employed in order to detect the PSP. For each line of the DIC grid, the position of this lower limit value was detected. The column and the line indexes were then used to evaluate the line equation of this PSP via a least mean square regression. As a result, the primary shear angle between the PSP and the free surface was calculated (normally horizontal).

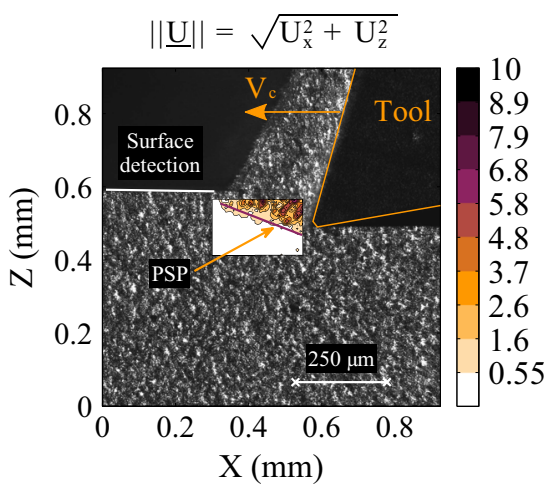

Fig. 7 Displacement field in primary shear zone for automatic measurement of shear angle
An automatic detection procedure for the unmachined surface was defined as follows in order to dispense with a possible tilting error of the free surface, which may have been induced by misorientation of the camera. A grey-level threshold was calculated out of the specimen based on the following criterion: $110 \%$ of the mean grey level value in a $200 \times 50$-px square region at the top left of the image (outside the workpiece). Fifteen strips of 50-px width and 2160-px height (the image height) equally distributed in the first half (from the left to the centre) of the picture were extracted. The mean values of the grey level over the 50 px for each pixel line were calculated and compared to the threshold. The mean values were found to exceed the threshold when the analysed pixel line was inside the material. Using the information on the unmachined surface position over the 15 strips, a linear least mean square regression was performed in order to estimate the surface tilt.

\section{Special Strain Calculation Procedure to Obtain Residual Strain Fields Induced by Orthogonal Cutting}

Particular attention was paid to the DIC as a result of a specific aspect related to cutting experiments, which is uncommon in mechanics: partial vanishing of the image corresponding to the removed material. If the ROI contained pixels within the removed material, an extremely large tension would be computed for the ZOIs containing this material, so as to satisfy the grey-level conservation principle. The DIC measurements would then be inaccurate. The use of the $\mathrm{P} 1 \mathrm{f0}$ frame as reference image did not allow the newly formed free surface limit to be estimated accurately. Therefore, the depth beneath the free surface for which the residual strains was not computed was increased. For the DIC, this adds the constraint that the P3f0 frame (after the cut) must be used as the reference frame, while the P1f0 frame (before the cut) must be used as the deformed frame, corresponding to $I$ and $J$ in Eq. (1), respectively. The $\mathrm{P} 3 \mathrm{f} 1$ and $\mathrm{P} 1 \mathrm{f} 1$ frames are also suitable for the calculation; therefore, the f0 index will no longer be mentioned. In this study, automatic detection of the free surface was used to guarantee that the selected ROI was inside the specimen, with $10 \mathrm{px}(6.6 \mu)$ remaining between the ROI and the top of the specimen. Thus, the residual strain was measured as closely to the machined surface as possible.

In order to be able to compare DIC results with postmortem measurements (residual stress, hardness), the state of interest has to be obtained after the cut, which corresponds to the mechanically deformed configuration. Last but not least, using the "post-cutting" pair as reference image allows to know the strain field over a regular mesh on deformed configuration that usually correspond to the post-mortem measurements. 
This explains why a special strain calculation method, presented in Fig. 8, was developed in order to express the machining induced strains $\left(\underline{\varepsilon}^{P 3 / P 1}\right)$ as functions of the depth beneath the machined surface. A direct calculation of the strains from the displacement field $\underline{U}^{P 1 / P 3}$ would lead to $\underline{\varepsilon}^{P 1 / P 3}$ (the strain applied in order to retrieve the initial state), which is inequivalent to the opposite of the strain applied from the initial state to the machined state $\underline{\varepsilon}^{P 3 / P 1}$.

As step 1, the DIC calculation (performed with Correli ${ }^{\mathrm{Q}} 4$ provided information on $\underline{U}^{P 1 / P 3}$, the displacement field to reshape the $\mathrm{P} 1$ image over the $\mathrm{P} 3$ image at the regular mesh coordinates $\left(X^{(3)}, Z^{3}\right)$. Then, the kinematic field at the mesh centres was calculated with Q4P1 interpolation over the regular grid. The deformed mesh $\left(X^{(1)}, Z^{(1)}\right)$ was computed as shown in Eq. (6). Thus, this calculation step corresponds to a "classical" DIC calculation of the field between the machined and unmachined specimens.

$\left(X^{(1) D}, Z^{(1 D}\right)=\left(X^{(3)}, Z^{(3)}\right)+\underline{U}^{P 1 / P 3}$.

As step 2, a regular mesh $\left(X^{(1)}, Z^{(1 I}\right)$ inside the deformed mesh $\left(X^{(1)}, Z^{(1 D}\right)$ was chosen with an element size of the same order as the original mesh. The nodal displacements of this regular mesh were calculated using a T3P1 shape function interpolation of the displacement field $\underline{U}^{P 3 / P 1}=-\underline{U}^{P 1 / P 3}$ at each node of the deformed mesh. The use of these shape functions guaranteed an interpolation with the same linear kinematic basis as for the DIC. Thus, there was no increase in the numerical noise induced by the DIC calculation during this numerical procedure. Mathematically, this process is conducted as follows: Each deformed cell $\left[M_{1}^{(1)}, M_{2}^{(1)}, M_{3}^{(1 D}, M_{4}^{(1)}\right]$ is decomposed into four triangles $\left(T_{1}, T_{2}, T_{3}, T_{4}\right)$ by the centre of the cell $C_{1}^{(1)}$. The displacements at $M_{i=1 \cdots 4}^{(1) D}$ and $C_{1}^{(1)}$ are known from the previous step. For each interpolated point $M^{(1 I}$, the containing triangle is selected and the displacements are calculated using the three corner displacements with the T3P1 shape functions. The displacement field $\underline{U}^{P 3 / P 1}$ is now known over a regular mesh. Thus, the strain field knowledge at each node of $\left(X^{(1)}, Z^{(1 I}\right)$, from the unmachined state is obtained in a straightforward manner through simple differentiation of the displacements.

Finally as step 3, a Q4P1 interpolation of the strain field at the deformed mesh centres $\left(C_{1}^{(D)}\right)$ is computed in order to map the strain fields at the initial mesh centres.

To draw a clear representation of the strain field induced by the machining operation, this field is overlaid on the image after the cutting operation (P3f0) (step 4).
Fig. 8 Protocol for residual strain field calculation in order to manage cut-induced partial vanishing of material

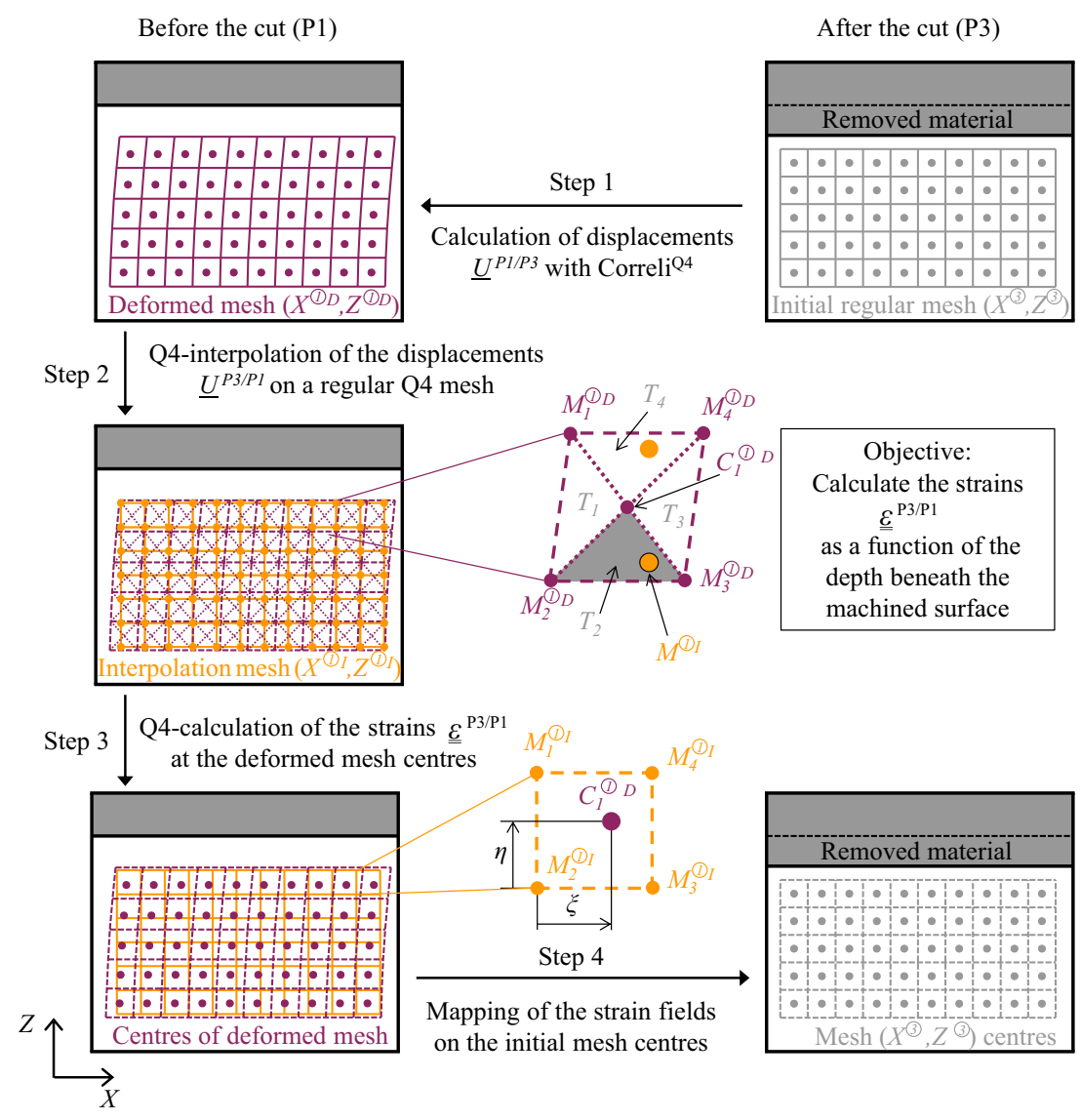


Table 4 Comparison between primary shear angles that were automatically detected, measured from the line between the root of the chip and the tool tip for 10 analysed tests, and predicted using the Merchant and the Lee and Shaffer models

\begin{tabular}{lll}
\hline & \multicolumn{2}{l}{ Shear angle } \\
\cline { 2 - 3 } PSP detection & $\begin{array}{l}\text { Mean values } \\
\left({ }^{\circ}\right)\end{array}$ & $\begin{array}{l}\text { Standard deviations } \\
\left({ }^{\circ}\right)\end{array}$ \\
\hline Automatic & 29.6 & 6.3 \\
Shear plane & 28.3 & 3.9 \\
Models & & \\
Merchant & 38.1 & 0.3 \\
Lee and Shaffer & 31.1 & 0.8 \\
\hline
\end{tabular}

\section{DIC Results and Repeatability Relative to Cutting Process}

The aim of this section is to present the characteristics relative to the orthogonal cutting operation, which can be measured via DIC using the experimental device and the proposed numerical methods.

\section{Primary Shear Zone Characteristics}

The automatic shear angle measurement procedure based on DIC presented in "Automatic Primary Shear Angle Measurement" was applied to the 1-bar micro-blasted surfaces. Further, the usual method for shear angle measurement as defined in the Merchant Theory (shear plane) was employed by measuring the angle formed by the line from the unmachined surface to the tool tip along the root of the chip. The results obtained from both methods are presented in Table 4 as mean values and standard deviations from the analysis of 10 image pairs. In addition, using the mean measured forces (cf. Table 5) and the tool geometry, it is possible to compute the Merchant [28] model and Lee and Shaffer [29] model predictions for the shear angle $\phi$. Both methods for shear angle determination (automatic and shear plane) yield a lower value than those predicted by the two models. The hypothesis in which non curling of the chip is assumed in these models may be the source of the obtained disparity. Those conclusions were also found by Sutter [10].

Table 5 Cutting force mean values and standard deviations for 10 trials per unit length of rectilinear edge

\begin{tabular}{lll}
\hline Forces/b & $\begin{array}{l}\text { Mean values } \\
(\mathrm{N} / \mathrm{mm})\end{array}$ & $\begin{array}{l}\text { Standard deviations } \\
(\mathrm{N} / \mathrm{mm})\end{array}$ \\
\hline$f_{c}$ & 87.7 & 1.2 \\
$f_{D}$ & 21.6 & 1.5 \\
\hline
\end{tabular}

\section{Displacement Fields Induced by Cutting Process}

First, a 5-px maximum deviation was found for the tool repositioning by detecting the Vickers indent formed on the side of the tool beforehand. This result demonstrates the good performance of the triggering device. However, to increase the analysis performance, the 10 repetitions on the sample prepared via 1-bar micro-blasting were resized in order to place the tool in precisely the same position in the images.

Then, the displacement fields induced by the cutting process could be measured by performing DIC between the P2 and $\mathrm{P} 1$ picture pairs with $l=12 \mathrm{px}$. The mean displacement fields and the standard deviation fields in both the $\mathrm{x}$ - and z-directions at each mesh node were computed and are presented in Fig. 9. Figure 9(a) and (b) show the mean fields of the 10 repetitions performed under identical conditions. The smooth shapes of the fields were well measured and resulted from the elastically behaving part deep inside the specimen as show in [30]. From analysis of the standard deviation maps (Fig. 9(c) and (d)), it is apparent that this part of the field is repeatable. When considering areas closer to the tool tip and the primary shear plane, the standard deviation increases showing less repeatable kinematic fields. This can be explained by the observed variation of the PSP angle between trials that modifies locally the boundary conditions (see "Primary Shear Zone Characteristics").

Finally, below the cutting edge, a layer of a few dozen microns exhibits a large deviation, which can be explained by considering a plastically deformed layer (that localised arbitrary) that is characteristic of the surface integrity engendered by the cutting process.

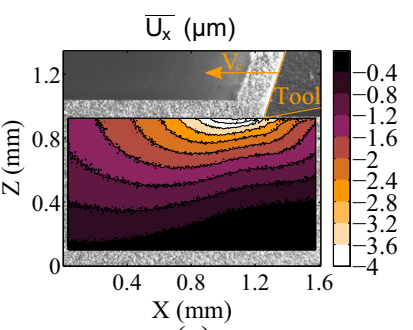

(a)

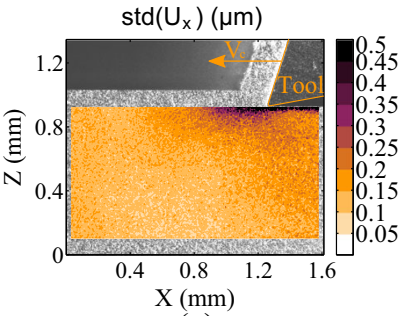

(c)

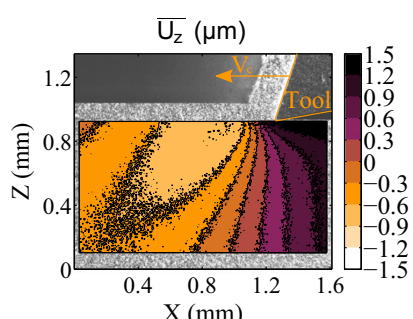

(b)

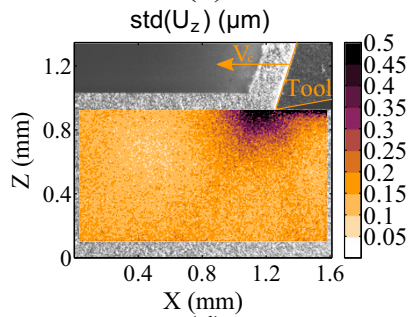

(d)
Fig. 9 Displacement fields induced during cutting process $\left(V_{c}=90\right.$ $\mathrm{m} / \mathrm{min}, \mathrm{h}=0.1 \mathrm{~mm}$ ) of 1-bar micro-blasted sample: Mean values in (a) $\mathrm{x}$ - and (b) z-directions; Standard deviations in (c) $\mathrm{x}$ - and (d) $\mathrm{z}$ directions. All results are for 10 repetitions 


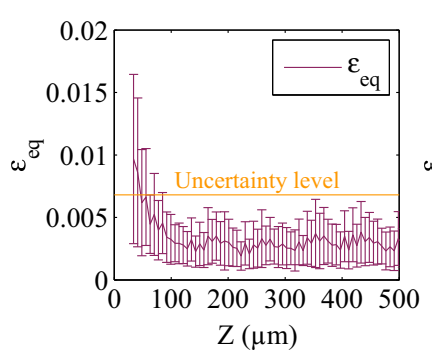

(a)

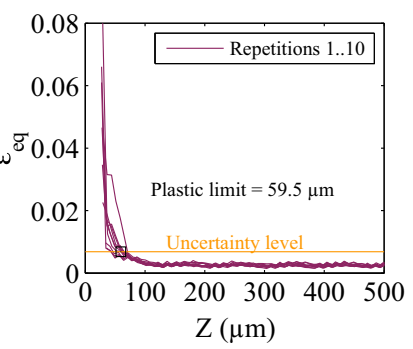

(b)
Fig. 10 Equivalent strain generated by cut versus depth (1-bar blasted surface): a Mean value and standard deviation along z-axis of $\varepsilon_{e q}$ field for repetition 1; b Line-by-line mean values of repetitions 1 to 10 and plastic limit determination

\section{Residual Strain Fields Resulting from the Cut}

The numerical procedure proposed in "Special Strain Calculation Procedure to Obtain Residual Strain Fields Induced by Orthogonal Cutting" was applied to the 1-bar sample over the 10 repetitions in order to determine the equivalent residual strain field $\varepsilon_{e q}$ in the material after the cut as calculated in [31]. Knowledge of the strain field at the centre of the regular mesh $\left(X^{3}, Z^{3}\right)$, allows the mean values of the field to be considered line-by-line as shown in [27]. The mean value and the standard deviation of the equivalent strain field along the z-direction (line-by-line) are shown in Fig. 10(a). By considering the previously determined DIC uncertainty ("Measurement Uncertainties"), the equivalent strain uncertainty was calculated using the error propagation. The $0.088-\mu \mathrm{m}$ displacement uncertainty induced a 0.006 uncertainty on the equivalent strain $\left(\varepsilon_{e q}\right)$. Thus, the equivalent plastic strain calculated inside the specimen was of the order of the uncertainty. The error bars, showing two times the standard deviation, remained below the uncertainty level of the equivalent plastic strain calculation.

Figure 10(b) shows the mean value of the equivalent strain field with respect to the depth beneath the machined surface for the 10 repetitions. Good repeatability of the aluminium machining process was obtained from the perspective of the surface integrity, as shown by the closeness of the curves. Thus, the plastically affected depth can be measured as being approximately $60 \mu \mathrm{m}$, in the present case, by detecting the depth before which the equivalent strain is higher than the uncertainty.

\section{Conclusions and Outlooks}

An experimental set-up for monitoring orthogonal cutting tests using DIC was demonstrated. Particular attention was paid to qualifying and setting the lighting device and the image scaling. Using a linear driven milling machine, cutting tests with rates of up to $120 \mathrm{~m} / \mathrm{min}$ can be achieved (limited to $90 \mathrm{~m} / \mathrm{min}$ in the present study). A specially developed triggering device allowed repetitions to be performed easily and with great repeatability (less than $4-\mu \mathrm{m}$ deviation of the tool position in the images).

Five surface preparations for examination via DIC were prepared and compared: the revealed microstructure (usually employed in machining) and four surfaces textured via micro-blasting at different pressures. Although the five specimens subjected to different preparation procedures did not exhibit differences in terms of the correlation radius criterion, the 1-bar blasted surface exhibited the lowest uncertainty considering the 30 repetitions were performed on the same area. The study of the DIC accuracy confirmed the choice of $S_{p d}$ and $S_{p c}$ as indicators of the future quality of the surface preparation. A high peak density $\left(S_{p d}\right)$ with low curvature $\left(S_{p c}\right)$ is preferred. Furthermore, the 1-bar surface exhibited the highest homogeneity throughout the specimen, whereas the etched specimen exhibited the lowest homogeneity. As a result of currying, the material texture was thus oriented leading to heterogeneity of the DIC. The preliminary analysis estimates an uncertainty of less than $0.1 \mu \mathrm{m}$ on the displacement fields.

After having proposed a primary shear angle detection procedure, results were compared to commonly used models for this angle prediction, namely, the Merchant and the Lee and Shaffer models. These models overestimated the shear angle compared to the experimental tests.

Then, the displacement fields induced during the cutting process were investigated by comparing image pairs taken before and during the cut. Significant repeatability of the engendered fields in the material was observed during the aluminium alloy machining. The obtained fields can be used to further material law identification via numerical simulations.

Finally, a special procedure for residual strain calculation was developed in order to manage the removed material, which contradicts the grey level conservation principle of the DIC. The equivalent strain maps were drawn and a $0.006-\varepsilon_{e q}$ uncertainty level was estimated. This level was employed to detect the plastic layer remaining in the material at a depth of approximately $60 \mu \mathrm{m}$.

Contrary to recently reported studies, particular attention was paid to the subsurface with reduced emphasis on the chip. The kinematic fields in the material during the cut are direct data that can be compared to numerical simulations. In the near future, both the macroscopic data (cutting forces, shear angle, chip thickness) and the local data (recorded kinematic fields) will be employed to validate a multiphysics cutting model. Knowledge of the thermal field produced in the material will also be invaluable for improvement of the simulation accuracy. 
Acknowledgments The authors acknowledge the Institut Carnot ARTS for their financial support through the UsiCorSurf project. They also gratefully thank ADEME and NTN-SNR for their support through the WindProcess project.

\section{References}

1. Moussaoui K, Mousseigne M, Senatore J, Chieragatti R (2015) The effect of roughness and residual stresses on fatigue life time of an alloy of titanium. Int J Adv Manuf Technol 78(1):557-563

2. Gravier J, Vignal V, Bissey-Breton S (2012) Influence of residual stress, surface roughness and crystallographic texture induced by machining on the corrosion behaviour of copper in salt-fog atmosphere. Corros Sci 61:162-170

3. Denkena B, Grove T, Dittrich MA, Niederwestberg D, Lahres M (2015) Inverse determination of constitutive equations and cutting force modelling for complex tools using Oxley's predictive machining theory. Procedia CIRP 31:405-410

4. Calamaz M, Coupard D, Girot F (2008) A new material model for $2 \mathrm{~d}$ numerical simulation of serrated chip formation when machining titanium alloy Ti-6Al-4V. Int J Mach Tools Manuf 48(3-4):275-288

5. Mabrouki T, Girardin F, Asad M, Rigal JF (2008) Numerical and experimental study of dry cutting for an aeronautic aluminium alloy (A2024-T351). Int J Mach Tools Manuf 48(11):1187-1197

6. Chen G, Li J, He Y, Ren C (2014) A new approach to the determination of plastic flow stress and failure initiation strain for aluminum alloys cutting process. Comput Mater Sci 95:568-578

7. Wright PK, Horne JG, Tabors D (1979) Boundary conditions at the chip-tool interface in machning: comparison between seizure and sliding friction. Wear 54:371-390

8. Poulachon G, Moisan A (2000) Hard turning: Chip formation mechanisms and metallurgical aspects. J Manuf Sci Eng 122(3):406-412

9. Childs THC (1971) A new visio-plasticity technique and a study of curly chip formation. Int J Mech Sci 13(4):373-387

10. Sutter G (2005) Chip geometries during high-speed machining for orthogonal cutting conditions. Int J Mach Tools Manuf 45(6):719726

11. Pujana J, Arrazola PJ, Villar JA (2008) In-process high-speed photography applied to orthogonal turning. J Mater Process Technol 202(1-3):475-485

12. Hijazi A, Madhavan V (2008) A novel ultra-high speed camera for digital image processing applications. Meas Sci Technol 19(8):085503

13. Arriola I, Whitenton E, Heigel J, Arrazola PJ (2011) Relationship between machinability index and in-process parameters during orthogonal cutting of steels. CIRP Ann Manuf Technol 60(1):93-96

14. List G, Sutter G, Bi XF, Molinari A, Bouthiche A (2013) Strain, strain rate and velocity fields determination at very high cutting speed. J Mater Process Technol 213(5):693-699
15. Pottier T, Germain G, Calamaz M, Morel A, Coupard D (2014) Sub-millimeter measurement of finite strains at cutting tool tip vicinity. Exp Mech 54:1031-1042

16. Sutton MA, Wolters WJ, Peters WH, Ranson WF, McNeill SR (1983) Determination of displacements using an improved digital correlation method. Image Vis Comput 1(3):133-139

17. Sutton MA, Orteu JJ, Schreier HW (2009) 5 Digital image correlation (DIC), Image Correlation for Shape, Motion and Deformation Measurements: Basic Concepts, Theory and Applications. Springer, US, pp 81-118

18. Bornert M, Hild F, Orteu JJ, Roux S (2012) 6 Digital Image Correlation. Wiley, pp 157-190. doi:10.1002/9781118578469.ch6

19. Besnard G, Hild F, Roux S (2006) Finite-Element displacement fields analysis from digital images: Application to Portevin - Le Châtelier bands. Exp Mech 46(6):789-803

20. Hild F, Roux S (2012) Comparison of local and global approaches to digital image correlation. Exp Mech 52(9):1503-1519

21. Ghadbeigi H, Bradbury SR, Pinna C, Yates JR (2008) Determination of micro-scale plastic strain caused by orthogonal cutting. Int J Mach Tools Manuf 48(2):228-235

22. Ghadbeigi H, Pinna C, Celotto S (2012) Quantitative strain analysis of the large deformation at the scale of microstructure: Comparison between digital image correlation and microgrid techniques. Exp Mech 52(9):1483-1492

23. Hanson RK (1988) Planar laser-induced fluorescence imaging. J Quant Spectrosc Radiat Transf 40(3):343-362. special Issue on Quantitative Spectroscopy and Laser Diagnostics

24. Peterson PD, Mortensen KS, Idar DJ, Asay BW, Funk DJ (2001) Strain field formation in plastic bonded explosives under compressional punch loading. J Mater Sci 36(6):1395-1400

25. Zhang D, Zhang XM, Xu WJ, Ding H (2016) Stress field analysis in orthogonal cutting process using digital image correlation technique. J Manuf Sci Eng. doi:10.1115/1.4033928

26. Baizeau T, Campocasso S, Fromentin G, Rossi F, Poulachon G (2015) Effect of rake angle on strain field during orthogonal cutting of hardened steel with c-BN tools. Procedia CIRP 31:166171

27. Outeiro JC, Campocasso S, Denguir LA, Fromentin G, Vignal V, Poulachon G (2015) Experimental and numerical assessment of subsurface plastic deformation induced by OFHC copper machining. CIRP Ann Manuf Technol 64(1):53-56

28. Oxley PLB (1962) Shear angle solutions in orthogonal machining. Int J Mach Tool Des Res 2(3):219-229

29. Lee E, Shaffer B (1951) The theory of plasticity applied to a problem of machining. J Appl Mech 18(4):405-413

30. Baizeau T, Campocasso S, Rossi F, Poulachon G, Hild F (2016) Cutting force sensor based on digital image correlation for segmented chip formation analysis. J Mater Process Technol. doi:10.1016/j.jmatprotec.2016.07.016

31. Buchkremer S, Klocke F, Lung D (2014) Analytical study on the relationship between chip geometry and equivalent strain distribution on the free surface of chips in metal cutting. Int J Mech Sci 85:88-103 